\title{
Corela
}

Cognition, représentation, langage

3-1 | 2005

Vol. $3, n^{\circ} 1$

\section{Un autre point de vue sur have}

\section{Catherine Douay}

\section{OpenEdition}

\section{Journals}

Édition électronique

URL : http://journals.openedition.org/corela/488

DOI : $10.4000 /$ corela. 488

ISSN : 1638-573X

\section{Éditeur}

Cercle linguistique du Centre et de l'Ouest - CerLICO

\section{Référence électronique}

Catherine Douay, « Un autre point de vue sur have », Corela [En ligne], 3-1 | 2005, mis en ligne le 04 juillet 2005, consulté le 19 avril 2019. URL : http://journals.openedition.org/corela/488 ; DOI : $10.4000 /$ corela.488

Ce document a été généré automatiquement le 19 avril 2019

\section{(c) (i) (2)(2)}

Corela - cognition, représentation, langage est mis à disposition selon les termes de la licence Creative Commons Attribution - Pas d'Utilisation Commerciale - Partage dans les Mêmes Conditions 4.0 International. 


\title{
Un autre point de vue sur have
}

\author{
Catherine Douay
}

1 Notre but dans la présente étude est de mettre en évidence le statut invariant de have à travers ses différents emplois. L'analyse que nous proposons s'inscrit dans le cadre de la T.R.I. (Théorie de la Relation Interlocutive), fondée sur l'hypothèse que les formes grammaticales se définissent par rapport au formatage de la réception ${ }^{1}$. Partant du principe que le locuteur, avant de modaliser son énoncé, doit utiliser des formes qui lui permettent déjà d'être compris, nous concevons en effet les structures morphosyntaxiques comme des solutions à des problèmes inhérents à la communication elle-même. Nous considérerons donc que have marque une stratégie interlocutive spécifique. Cette stratégie qui, comme nous le verrons, permet au locuteur d'amener l'interlocuteur à considérer les implications contextuelles de la proposition, caractérise l'une des trois configurations de la relation interlocutive (c2) qui, dans notre modèle, informent toute l'architecture de la langue. Selon notre hypothèse, un énoncé avec have est donc fondamentalement prospectif. Qu'il déclare :

1. He has three cars.

2. John has been in London.

2 le locuteur met au sens propre l'interlocuteur devant "le fait accompli ". Il constate ce que Benveniste (1966) appelle "l'état de l'ayant", en occultant toute référence à l'origine de cet état. En (1), par exemple, il porte à l'attention de l'interlocuteur que le référent du sujet syntaxique " he" se trouve posséder trois voitures. En (2), il l'informe que "John" se trouve dans l'état "avoir séjourné à Londres". Dans les deux cas, il oriente l'interlocuteur sur les implications de ces états de "l'ayant". Nous verrons que cette thèse est loin de faire l'unanimité, de nombreuses analyses s'attachant au contraire à mettre en évidence la dimension " anaphorique " ou " présupposante " de have.

Selon une conception courante chez les linguistes rattachés au courant énonciativiste, have serait fondamentalement un marqueur d'aspect. Pour J. Bouscaren / J. Chuquet, par exemple, ilsignale que l'énonciateur "localise", "repère" le procès par rapport à l'élément promu au rang de sujet et ipso facto " thématisé ". Il s'agit donc de présenter le procès sous un certain angle, un certain aspect, autrement dit un certain point de vue. On 
entre ainsi avec have dans le domaine du subjectif: have est dit exprimer le "point de vue" de l'énonciateur. On retrouve cette notion de point de vue associée à have dans quelques analyses récentes sous le concept " d'empathie". Cette fois, il ne s'agit plus du point de vue de l'énonciateur mais de celui du référent du sujet syntaxique. Ainsi, pour C. Boisson (1987), have signale "empathiquement" que "tout l'énoncé est filtré par la subjectivité de l'élément qui occupe la place syntaxique de sujet". De même, P. Cotte (1997: 50) déclare que "l'énoncé avec have ne se contente pas d'un constat objectif : il introduit un point de vue". On adopte le "regard" du sujet syntaxique. Notre analyse interlocutive de have nous amènera à la conclusion inverse : les énoncés avec have sont explicitement détachés de tout point de vue particulier. Nous montrerons que c'est ce critère qui permet de les différencier des énoncés avec be, explicitement rattachés à un point de vue source.

\section{Cadre théorique : les trois configurations de la relation interlocutifve}

4 Rappelons pour commencer les grandes lignes de notre modèle théorique, fondé sur l'hypothèse qu'un système linguistique est organisé en prévision de ce à quoi il va servir et que les variables de discours sont organisées par les formes pour faire sens commun. Cette interdépendance langue/discours est loin d'être acceptée uniformément. La bipolarité du phénomène a en effet conduit à une bi-polarisation entre les théories fondées sur le postulat de l'autonomie du système et celles fondées à l'inverse sur le principe d'une subordination totale de la forme à l'usage et au contexte. Entre ces deux positions extrémistes, la pragmatique en général accepte comme "légitime" un divorce entre les deux plans et s'accommode d'une linguistique formelle du "code" qui traite des problèmes qu'elle ne peut pas aborder elle-même.

5 En continuant d'habiliter un statut spécifique pour le sujet parlant, comme pivot de la systématique, les théories de l'énonciation ont cherché à maintenir une analyse interactive : la langue, selon G. Guillaume, n'existe qu'en vue du discours. Certes, mais pour les énonciativistes, il s'agit avant tout de montrer que le locuteur s'inscrit dans l'énoncé, qu'il y imprime sa marque, son “point de vue". L'Autre est souvent aperçu, mais comme une sorte d'alter ego, qui n'intervient pratiquement dans l'analyse linguistique que pour l'étude des effets de sens " pragmatiques". La perspective demeure monologale. C'est l'énonciateur qui reste maître de la parole parce que c'est lui qui fonde la langue. En aucune façon, l'interlocuteur n'est conçu comme paramètre fondateur du dispositif langagier.

6 Ce privilège accordé au locuteur a été ces dernières décennies logiquement discuté par les tenants de l'approche interactionniste. La promotion d'une conception dialogale de l'acte de langage, en ouvrant à l'analyse linguistique un nouveau champ d'investigation, a permis l'exploration, qui se poursuit, de phénomènes passés sous silence dans les approches monologales. Il semble cependant abusif de parler, à propos des retombées théoriques de l'approche interactionniste sur la linguistique en général, de "mutation fondamentale ${ }^{2}$ ". La fermeture mutuelle des deux pôles n'est en effet pas remise en question : tout en insistant sur le fait que l'interaction constitue la réalité fondamentale du langage, les interactionnistes confortent l'hypothèse qu'il y aurait au fond un système de représentation linguistique déconnecté des problèmes relatifs à la communication. 
7 Pour notre part, nous défendons l'idée que les systèmes de langue sont structurés pour répondre aux besoins et contraintes de la communication: dans une problématique générale de l'émission et de la réception, c'est la réception qui est la clé de voûte des systèmes de langue. Aucune parole ne peut être conçue qu'elle ne soit construite pour être entendue et comprise. Les formes du message, qui intéressent le linguiste, résultent de ce besoin d'être compris d'autrui et le besoin ou la faculté de s'exprimer n'a de sens qu'à l'endroit d'un récepteur. Autrement dit, la langue a pour vocation d'apporter des solutions au problème de l'entente interlocutive : s'il est question de parler de choses et états de choses actuels ou virtuels, il s'agit pour les interlocuteurs de se mettre d'accord sur ce dont ils parlent. C'est aux conditions systématisées en langue de cette " mise en accord", ce que F.Jacques (1979) définit comme les "conditions a priori de la communicabilité ", que nous nous intéressons.

Précisons que si les interlocuteurs utilisent des formes de langue, ce sont des formes de codification de rôles linguistiques interactifs. Ces rôles sont universels et ne sont en aucune façon déterminés par les rôles et conditions psychologiques et / ou sociologiques des individus ou groupes empiriques. Cette confusion généralement entretenue en pragmatique est lourde de conséquences en ce qu'elle tend à réduire la linguistique à une sous-discipline de la sociologie. Pour éviter cette confusion, nous désignons respectivement les rôles locutif et allocutif par $\alpha$ et $\beta$, qui ne préjugent en rien de ces déterminations ${ }^{3}$.

Selon notre hypothèse, l'accord interlocutif peut être obtenu selon trois stratégies qui correspondent chacune à une configuration particulière de la relation entre les deux pôles allocutifs.

La configuration du Rapport Interlocutif Direct (R.I.D.)représente un premier mode, central, de résolution de l'écart "référentiel" entre les interlocuteurs. Cette configuration (Co) correspond à une situation où les représentations d' $\alpha$ et de $\beta$ sont censées pouvoir et devoir coincider immédiatement. Un accord de type RID est fondé sur des données situationnelles (au sens étroit ou large) évidentes ou réputées telles. Une forme RID, non ou minimalement marquée, signale systématiquement l'absence de besoin de marqueurs supplémentaires pour la réalisation de l'accord interlocutif.

11 Les deux autres configurations ( $\mathrm{C} 1$ et $\mathrm{C} 2)$ renvoient, elles, à une situation de décalage interlocutif (soit on ne peut pas, soit on ne veut pas fonctionner sur l'évidence situationnelle du R.I.D.) et à la nécessité corrélative de mettre en œuvre des stratégies spécifiques pour que la proposition entendue par $\beta$ soit identique à la proposition d' $\alpha$ malgré le décalage. La situation ne permettant plus comme en RID de s'appuyer sur une communauté de référence évidente, c'est sur la connivence que s'établit l'accord en C1 et C2. Autrement dit, les formes $\mathrm{C} 1$ et $\mathrm{C} 2$ signalent une nécessité de marquer, en vue de la réalisation de l'accord, ce qui en RID irait sans dire.

12 La stratégie mise en œuvre en $\mathrm{C} 1$ consiste à contourner, court-circuiter le décalage en invitant $\beta$ à se placer du point de vue $\alpha$ (se "mettre à la place " d' $\alpha$ ) - tout en conservant par ailleurs son propre point de vue, éventuellement contraire mais décrété sans incidence sur la validation de la proposition $\alpha$. Autrement dit, $C 1$ fonctionne sur une demande explicite de mise entre parenthèses de la dimension $\beta$ du rôle allocutif. Tout est ramené au point de vue $\alpha$, à la source, aux conditions initiales. $C 1$ entérine ainsi le décalage entre les deux pôles $\alpha$ et $\beta$ mais passe outre. Etant déclarée validable nonobstant les variations éventuelles de $\beta$, explicitement envisagées, la proposition $\alpha$ se trouve 
valorisée. Quelle que soit la spécificité du marqueur considéré, la personnalisation induite par $\mathrm{C} 1$ contraste toujours nettement avec l'impersonnalisation de $\mathrm{C} 2$. Selon l'hypothèse défendue ici, be est un marqueur $\mathrm{C} 1$ et have un marqueur $\mathrm{C2}$.

$\mathrm{C} 2$ fonctionne à l'inverse de $\mathrm{C} 1$ sur une demande explicite de mise entre parenthèses de la dimension $\alpha$ du rôle allocutif. C'est donc la stabilisation du pôle de réception $\beta$ (et non comme en $\mathrm{C} 1$ du pôle d'émission) qui permet l'obtention de l'accord. La connivence est ainsi obtenue par un effet de nivellement entre $\alpha$ et $\beta$ : la réception étant imposée commme définitivement acquise (corrélativement au gommage de la dimension $\alpha$ du rôle allocutif), la variation d' $\alpha$ devient elle-même nulle et non avenue. Les deux pôles $\alpha$ et $\beta$ se retrouvent ainsi en $\mathrm{C} 2$ contraints d'avaliser une proposition décrétée validée dans l'absolu (et non relativement à un point de vue source). Par contraste avec les marqueurs C1, marques du relatif, les marqueurs C2 peuvent donc se définir comme marques de l'absolu. Ils ont une valeur "conclusive" dans la mesure où la référence est posée et imposée comme un absolu, définitivement stabilisé, transcendant toutes les situations et à propos duquel il est déclaré impossible d'intervenir. C'est précisément en fermant l'accès à la proposition qu'ils obligent à en considérer la subséquence.

Les auxiliaires have et be, intercalés entre le sujet et le prédicat, ne sont donc pas pour nous interprétables comme marques d'une inscription modale de l'énonciateur. Ils renvoient à deux configurations différentes de la relation interlocutive, qui ont en commun de nous projeter hors RID, dans une situation de décalage interlocutif: l'association du prédicat au sujet syntaxique n'est plus donnée à entendre comme une relation immédiatement et simultanément accessible aux deux pôles de l'allocution. Ce que marque la présence d'un auxiliaire, c'est ce décalage interlocutif, plus exactement l'impossibilité de résoudre ce décalage en se fondant sur l'évidence de données situationnelles. Il s'agit donc de créer avec $\beta$ une connivence. Ce qui distingue be et have découle du mode de résolution du décalage ou, si l'on préfère, de la stratégie mise en œuvre pour établir cette connivence interlocutive. En C1, dont relève be, la demande explicite de mise entre parenthèses de la dimension $\beta$ du rôle allocutif a pour effet de renforcer la proposition $\alpha$ (décrétée validable nonobstant le décalage), tout en la relativisant. Have, marqueur $\mathrm{C} 2$, résout le décalage par une demande explicite de mise entre parenthèses du pôle source (aussi bien au niveau d' $\alpha$ que de $\beta$ ), figeant ainsi la relation prédicative dans l'absolu et contraignant de ce fait l'interlocuteur à se tourner vers les implications de la proposition.

Une assertion avec have se donne donc à entendre comme assertion explicitement détachée de tout point de vue particulier. Occultant toute référence au pôle locutif $\alpha$, have oriente le récepteur vers la subséquence de la proposition. Ce centrage sur la subséquence s'observe de façon très nette dans les formes verbales de parfait / perfect have + v-en ou have + been + V-ing. Ce qu'on désigne couramment par "l'état résultant" du sujet, sur lequel l'attention est attirée, n'est jamais l'objet explicite du message.

\section{Le parfait}

Considérons l'énoncé suivant analysé par J-C. Souesme ( 1992 : 72) :

3. I've washed the dishes.

Le present perfect have washed marque, selon l'auteur, un "état résultant ", à savoir que les assiettes, qui étaient sales, sont maintenant propres: il y a donc, souligne J.C. Souesme, "passage d'un état à un autre". Le procès à l'origine de l'état résultant est 
explicitement mentionné dans l'énoncé sous la forme du participe passé. Pour autant, la mise en relation $\mathrm{S} / \mathrm{P}$ a déjà été effectuée. D'où la conclusion que have est un "opérateur métalinguistique de rappel d'une relation prédicative déjà effectuée" (1992: 75). On retrouve pour l'essentiel la même analyse chez P. Cotte (1998): l'énoncé avec have "présuppose" une relation prédicative déjà effectuée, qui reste dans le préconstruit, l'implicite, l'attribution à un sujet de cette relation prédicative préconstruite (rappelée dans le cas du parfait sous la forme du participe passé) passant ainsi explicitement au premier plan.

18 Cette analyse suscite plusieurs interrogations, tout d'abord à propos de ce qu'est "l'état résultant". Il nous semble en effet difficile d'accepter que have washed marque le passage d'un état de saleté à un état de propreté. Tout ce que dit le locuteur, c'est qu'il se trouve avoir lavé les assiettes. Dans des circonstances normales, le bon sens peut effectivement nous amener à conclure que, si le sujeta lavé les assiettes, c'est parce qu'elles étaient sales. Mais rien n'interdit, dans d'autres circonstances, de penser qu'il se soit livré à cette activité pour une toute autre raison. Surtout, rien, hors contexte, ne nous autorise à conclure que c'est sur cet état de propreté des assiettes que have washed attire notre attention. L'état résultant n'est jamais explicitement l'objet de l'énoncé. C'est toujours à l'interlocuteur d'inférer cet objet, en fonction des différents indices dont il dispose. Il est d'ailleurs intéressant de noter que, tout en définissant have comme marqueur du point de vue de l'énonciateur (il s'agit pour l'énonciateur " d'exprimer sa position et de qualifier le sujet de l'énoncé ", op.cit. : 78), J.-C. Souesme observe, à propos de la comparaison entre

4. He builds houses.

5. He has built his house himself.

qu'avec le perfect, "l'énonciateur laisse en quelque sorte 4 à l'interlocuteur le soin de déduire la propriété, caractéristique ou état du sujet " (op. cit. : 76). Le " en quelque sorte" est très révélateur à nos yeux de la difficulté à théoriser la place de l'interlocuteur dans une approche énonciative et de la réticence corrélative à lui accorder la place qui est la sienne. Tout aussi révélatrice de cette réticence et de cet embarras nous apparaît la conclusion de J.-C. Souesme à la fin de son chapitre sur le present perfect :

“ en permettant de déduire une caractéristique ou un état du sujet, have + -en n'est pas une forme aussi innocente ${ }^{5}$ que le présent ou le prétérit même si les faits paraissent toujours présentés de manière objective. L'énonciateur invite néanmoins ${ }^{6}$ le co-énonciateur à en tirer un jugement de valeur personnel " (op. cit. : 78)

Pour revenir à (3), si, par exemple, des interlocuteurs se sont disputés à propos du partage des tâches ménagères au sein de leur foyer, l'énoncé - entendu avec le ton approprié et un regard 7 qui " en dit long " - pourra être interprété comme signifiant “ Tu ne pourras plus me reprocher de ne rien faire dans la maison". Si le participe passé "washed " évoque bien un fait tenu pour acquis, have a lui une valeur fondamentalement prospective et non présupposante ou anaphorique : il oblige l'interlocuteur à s'intéresser aux implications de la proposition, il est tout entier tourné vers la subséquence, le futur. Nous maintenons donc qu'en déclarant (3) I've washed the dishes, le locuteur dit qu'il se trouve dans l'état d'avoir lavé les assiettes, à l'interlocuteur de s'orienter sur "la suite à donner " à cet " état de fait " " bonne chose, les assiettes sont propres " ou " c'est bien, tu as fait un effort pour le ménage ", etc). Have n'a pas pour fonction première d'exprimer le point de vue personnel de l'énonciateur, ni le point de vue du référent du sujet syntaxique dont l'énonciateur adopterait "empathiquement ${ }^{8}$ " le regard. Si un point de 
vue est en cause, ce ne peut être que celui de l'interlocuteur qui, n'ayant aucune prise sur la relation prédicative elle-même, est contraint alors de s'intéresser à ses implications.

Have garde la même valeur lorqu'il est suivi de been $+-V$-ing : il oriente l'interlocuteur sur les implications de la proposition en be $+V$-ing qui, à la différence du participe passé énonçant un simple constat détaché du locuteur, est donnée à entendre comme proposition explicitement rattachée au locuteur (soit que ce dernier identifie pour l'interlocuteur une relation prédicative dont la nature n'est pas "évidente", soit qu'il porte à sa connaissance une information dont il souligne qu'il en est le seul détenteur, soit encore qu'il donne sa propre interprétation, appréciation, des faits). Comme nous l'avons expliqué, la stratégie définitoire de $\mathrm{C} 1$ fonctionne sur un décalage interlocutif déclaré ne pas faire obstacle à la validation, alors qu'en C2 la validation est obtenue par un nivellement des deux pôles. La variété des effets de sens du parfait en be $+V$-ing découle donc de la variété des effets de sens de be $+V$-ing et non d'un sens particulier que prendrait have en combinaison avec cette forme. Simplement à titre d'exemple, examinons brièvement l'énoncé ci-dessous :

6. Cutting through the lanes from the main road to Basingstoke, Diana Jago passed an unremarkable car unremarkably parked in a gateway. [...] It was only when Diana was twenty yards past this car that she realized that the man in it had been neither eating nor sleeping. He had been staring in front of him in a most unnatural way. (A Passionate Man, J. Trollope, 1990)

Les trois phrases où figure have + been $+V$-ing, tout en étant centrées sur l'état du référent du sujet syntaxique " he ", valorisent l'interprétation de l'énonciateur - ici le personnage Diana dont la délibération intérieure nous est rapportée indirectement. La locutrice remet elle-même en cause sa première interprétation : contrairement à ce qu'elle avait d'abord cru, ce n'est pas "eat" ou "sleep" que faisait le personnage mais "stare in a most unusual way". Pour autant, et contrairement à ce qui se passerait avec une forme dite progressive sans "have" ("he was staring in a most unusual way"), le narrateur, plutôt que d'inviter le lecteur à s'attarder sur le prédicat retenu, l'oriente d'emblée sur les implications contextuelles de cet état du sujet réinterprété. Ce que confirme la suite du texte puisque le personnage Diana décide de faire demi-tour pour aller porter secours au référent de " he ". La proposition en be + V-ing, bien que valorisée en tant que proposition inédite, en décalage avec un point de vue autre (ici, qui avait été explicitement envisagé et évoqué), se trouve donc avec have reléguée à l'arrière-plan interlocutif dans la mesure où la question n'est pas tant de s'interroger sur l'exactitude de l'analyse du locuteur que d'inviter l'interlocuteur à considérer les implications. La mention explicite et fréquente (dans les cotextes des parfaits en - ing) des indices sur lesquels s'appuie le locuteur pour “justifier " sa proposition peut être interprétée comme allant dans le même sens, à savoir comme moyen de court-circuiter en quelque sorte la dimension "polémique", "délibérative" de be $+V$-ing: la proposition étant légitimée par un certain nombre d'indices, elle s'expose moins à la réfutation, ce qui contribue à reporter toute l'attention de l'interlocuteur, comme le prescrit have, sur les implications contextuelles de la proposition (que be rattache explicitement à un point de vue source). La richesse des effets de sens du perfect en $V$-ing demanderait bien entendu une étude à part entière. Nous avons simplement voulu ici mettre en évidence l'invariant de have quels que soient les éléments avec lesquels il entre en combinaison.

L'interlocuteur étant contraint avec have de s'intéresser aux implications contextuelles de la proposition, on peut dire que les formes de parfait ont pour effet de mettre au premier 
plan "l'incidence présente" du procès plutôt que le procès en lui-même (théorie de la "current relevance "). Mais cette valeur découle selon nous de la stratégie interlocutive caractéristique de $\mathrm{C} 2$ dont relève have. Par ailleurs, la théorie de la "current relevance " omet un élément pour nous essentiel, à savoir que ni have ni $-E N$ ne donnent en euxmêmes d'indication sur la nature exacte de l'incidence présente du procès qu'il incombe à l'interlocuteur de déterminer. D'autres grammairiens mettent en avant la dimension "explicative" des formes de parfait: mentionner explicitement, par le recours au participe passé, le "procès à l'origine de l'état résultant ", revient à expliquer l'état en question. Cette thèse va à l'encontre de notre propre analyse qui met en avant l'absence avec have de toute dimension explicative : le locuteur constate, avons-nous dit, l'état de l'ayant, en occultant toute référence aux causes initiales. L'état résultant n'étant jamais évoqué explicitement, il n'est pas possible d'établir un lien de causalité directe avec le procès évoqué au participe passé. Pour autant, nous ne nions pas qu'un énoncé avec une forme de parfait puisse s'incrire dans une relation de causalité mais nous maintenons que have $+V$-en n'a en soi aucune valeur explicative. En disant :

7. He has not had breakfast.

le locuteur porte à l'attention de son interlocuteur " l'état de l'ayant", à savoir l'état du référent de "he " qui, pour une raison non évoquée (soit que le locuteur l'ignore ou que cela n'a aucune importance à ses yeux), se trouve dans la situation " not had breakfast " (ne pas avoir pris de petit-déjeuner). Orienté d'emblée, par le truchement de have, sur les implications de la proposition, l'interlocuteur, guidé par la recherche de pertinence, va, en fonction de la situation et des indices élocutionnels, "faire sens" de cet état de fait porté à son attention. Il peut, par exemple, être amené à conclure que le référent de " he " a faim et qu'il faut lui donner à manger. En termes d'analyse dite "logique ", ceci revient, certes, à établir une relation de causalité, l'énoncé avec have + V-en devenant dans cet enchaînement "cause" de la faim présente du référent de "he" et de la nécessité corrélative de le nourrir. Mais cette valeur explicative, causative, n'est que la résultante d'une inférence contextuelle de l'interlocuteur qui, pour rendre l'énoncé pertinent, est contraint de le "rattacher" à autre chose. Tout au plus peut-on dire que have, par son orientation de l'interlocuteur sur les implications contextuelles de la proposition, favorise en quelque sorte par défaut la recherche de liens avec d'autres éléments en dehors de l'énoncé lui-même.

De même, seule une inférence contextuelle permet d'attribuer une valeur causative à have dans un énoncé du type :

8. I had my car stolen.

Comme le soulignent J. Bouscaren et J. Chuquet (1982: 62), à qui cet exemple est emprunté, (8) “ peut supporter les deux interprétations selon la situation dans laquelle il est prononcé : causative (j’ai organisé le vol) ou de thématisation (voilà ce qui m'est arrivé)". Have ne marque en effet aucune relation de causalité. Orientant sur la subséquence, il engage l'interlocuteur à inférer ce qui est impliqué par le message et à possiblement évaluer, ipso facto, le degré d'agentivité du référent du sujet syntaxique.

Pour résumer, nous poserons que, de façon générale, une assertion avec have est donnée à entendrecomme assertion catégorique, valide quels que soient les circonstances et les interlocuteurs (variation du pôle source décrétée indifférente, réception définitivement acquise). 


\section{Have lexical et have to $V$} have est explicitement détaché de tout point de vue particulier, va à l'encontre de la plupart des théories existantes, qui analysent have comme marqueur de " point de vue". Des divergences importantes apparaissent cependant quant à l'origine de ce point de vue. Pour J.-C. Souesme, nous l'avons vu précédemment, il s'agit du point de vue de l' énonciateur (" have permet à l'énonciateur d'introduire son point de vue personnel sur l'état du sujet", 1992 : 77). Pour C. Boisson (1987), c'est au point de vue du référent du sujet syntaxique, dont le locuteur adopte "empathiquement" le regard, que renvoie have (nous y reviendrons). De même, à propos de (9) ci-dessous, P. Cotte (1997: 50) déclare que "l'énoncé en have ne se contente pas d'un constat objectif. Il introduit un point de vue : Connie [she] a conscience de son abandon et have marque l'empathie: on adopte son regard":

9. He held her fast and she felt his urgency. Her old instinct was to fight for her freedom. But something else in her was strange and inert and heavy. His body was urgent against her, and she hadn't the heart anymore to fight. He looked around. 'Come - come here! Through here', he said, looking penetratingly into the dense fir-trees [...]. He looked back at her. She saw his eyes, tense and brilliant, fierce, not loving. But her will had left her. A strange weight was on her limbs. She was giving way. She was giving up. (Lady Chatterley's Lover, D.H. Lawrence, 1928).

Nous sommes d'accord qu'il existe une différence entre l'énoncé en have [she hadn't the heart anymore to fight] et l'énoncé en be que P. Cotte lui compare (énoncé qui figure également dans le texte dont est extrait le passage cité) :

10. There wasn't any courage left in her to fight.

Cependant, il nous semble difficile d'en rendre compte dans les termes de l'auteur. Le narrateur verbalise, certes, un sentiment éprouvé par son personnage Connie, ce qui suppose qu'il se projette dans l'univers mental de cette dernière, en d'autres termes, qu'il " adopte son regard". Mais dans tout le paragraphe, le narrateur nous transmet, par la technique du discours indirect libre, des sentiments et sensations éprouvés par Connie, dont celle-ci a (plus ou moins) conscience. En d'autres termes, tout, dans l'extrait cité, est filtré par le regard du personnage. Pour autant, have n'est pas systématiquement utilisé, comme en témoignent les trois dernières phrases de l'extrait qui continuent à nous faire entendre Connie avec cette fois be. On notera en particulier le recours à be $+V$-ing pour évoquer la conscience qu'elle a de s'abandonner [She was giving way. She was giving up]. De même, A strange weight was on her limbs verbalise une sensation éprouvée par le personnage (et dont rien dans le texte ne nous autorise à considérer qu'il s'agirait cette fois d'une sensation dont elle ne serait pas consciente), ce qui laisserait attendre, si l'on suit la démonstration de P. Cotte, She had a strange weight on her limbs.

Nous maintenons donc que have ne peut en lui-même permettre de "localiser" les points de vue exprimés, d'ancrer avec certitude les regards. Le narrateur nous a installés dès le début dans l'univers intérieur du personnage et c'est ce ce qui nous amène à intégrer spontanément à cet univers les pensées et sensations formulées. Pour revenir à (9) She hadn't the heart anymore to fight, le narrateurporte la parole de Connie qui constate l'état dans lequel elle se trouve et auquel, plus exactement aux "implications" duquel elle est en fait déjà résignée. Tout le texte établit en outre clairement qu'elle ne comprend pas elle-même cet état d'impuissance dans lequel elle se trouve face à " he " (cf. notamment la

Corela, 3-1 | 2005 
répétition de strange). Nous retrouvons donc dans cet exemple ce qui, selon notre hypothèse, constitue la valeur fondamentale de have : l'absence de dimension explicative (interlocuteur - ici Connie elle-même - mise devant le "fait accompli ") et la focalisation corrélative sur la suite de la proposition. Les trois énoncés en be $(+V$-ing) nous livrent quant à eux l'analyse introspective à laquelle se livre le personnage dans ce qui peut être interprété comme une tentative désespérée pour ne pas perdre tout contrôle de la situation qui, de fait, lui échappe complètement. A strange weight was on her limbs témoigne ainsi d'un effort pour tenter d'analyser lucidement, d'expliquer, dans son for intérieur, ce qu'elle ressent alors que she had a strange weight on her limbs la montrerait subissant ce poids étrange qu'elle ne s'expliquerait pas (ou ne chercherait pas à s'expliquer) mais qu'elle assumerait, prête à se donner à " he " (focalisation sur les implications). Grâce à la superposition du dialogue intérieur du personnage avec elle-même et du dialogue narrateur/lecteur, rendue possible par la technique du discours indirect libre, have dans she she had a strange weight on her limbs créerait en même temps une attente chez le lecteur, orienté par le truchement de have sur la suite de la narration. La succession des trois énoncés avec be (A strange weight was on her limbs; She was giving way. She was giving up) montre que le narrateur a préféré d'abord inviter son lecteur à s'attarder sur l'analyse de l'état dans lequel se trouve le personnage.

Cet exemple confirme que be et have, en orientant l'interlocuteur dans des directions différentes, jouent un rôle essentiel dans la stratégie interlocutive et que c'est par rapport à ces questions de stratégie interlocutive - qui, selon notre hypothèse, informent toute la systématique de la langue - qu'ils doivent être définis. L'orientation de l'interlocuteur sur la suite de la proposition permet d'expliquer un autre emploi très courant de have illustré par les exemples ci-dessous empruntés à C. Boisson (1987) :

11. We have this girl I'm sure I told you who chooses the head words for the dictionary.

12. I have a cousin his name is Jack.

Comme le souligne à juste titre C. Boisson au début de son article (Anglais 'have', français 'avoir' et l'empathie, 1988), cet emploi de have est peu étudié alors qu'il s'agit d'un emploi très courant, qu'on retrouve en français avec avoir :

13. J'ai mon frangin, côté nanas, ça va pas fort en ce moment.

L'auteur s'attache d'abord à démontrer que la définition culiolienne d'avoir comme marqueur de thématisation ${ }^{9}$ est insuffisante pour rendre compte de cet emploi. Nous ne retiendrons ici que l'argument jugé décisif par l'auteur pour justifier sa thèse. Dans (13) ci-dessus, le thème serait non pas "Je "mais "côté nanas", ce qui amène $\mathrm{C}$. Boisson à conclure qu'avoir n'a pas pour fonction de thématiser le sujet syntaxique (cette fonction étant dévolue à "côté nanas"). Il permettrait une "empathisation sur le sujet syntaxique".

Outre le fait que l'empathie sur le sujet syntaxique nous paraît très difficile à déceler dans de nombreux exemples évoqués par C. Boisson, l'analyse du rôle de "côté nanas" nous semble très discutable. En (13), "côté nanas" permet au locuteur de signaler explicitement qu'il limite son évocation de la situation de son frère au domaine des femmes (" côté nanas", sous-entendu, par exemple : "je ne parle pas du reste où, là, tout va très bien " ou au contraire : "où, là, ça va encore plus mal "). En d'autres termes, " côté nanas" opère une "focalisation contrastive", ce qui est très différent du rôle de "j'ai". Pour autant, nous sommes d'accord avec C. Boisson que la définition d'avoir (transposable 
à have) comme opérateur de thématisation ne permet pas de rendre compte de façon satisfaisante de la fonction du segment " $\mathrm{j}$ 'ai mon frangin ".

On notera que tous les énoncés du corpus de C. Boisson sont isolés de leur cadre conversationnel, ce qui tend de façon générale à occulter le problème de la fonction discursive, plus précisément dialogale, des formes soumises à l'analyse. Alors même que l'auteur précise que la plupart des exemples de son corpus sont extraits de conversations d'enfants ou d'adultes, les énoncés ne sont jamais présentés dans le cadre de l'échange interlocutif auquel ils participent. Or, si l'on s'interroge sur le rôle de “j'ai mon frangin " en (13) dans la machinerie conversationnelle, il apparaît qu'il permet avant tout au locuteur d'introduire le topique de la conversation (à savoir son frère) en évitant de le promouvoir au rang de sujet syntaxique. On imagine en effet difficilement, dans une conversation ordinaire, le locuteur annonçant de but en blanc à son interlocuteur :

14. Mon frangin ne va pas fort en ce moment, côté nanas

Cette fonction phatique d'accroche de J'ai (transposable ici à I have, $c f$. exemples 11 et 12), qui permet au locuteur de "prendre la parole ", i.e. d'annoncer à l'interlocuteur qu'il va lui dire quelque chose, découle selon nous des caractéristiques de $\mathrm{C} 2$ dont relève have. Il s'agit de "forcer l'écoute" de l'autre et la valeur prospective de have le rend particulièrement apte à remplir cette fonction.

La différence entre be et have s'observe également de façon très nette dans les formes dites périphrastiques have to vs is to. Notons tout d'abord que la non-agentivité du sujet de have et de be, verbes d'état, permet d'expliquer l'affinité de ces deux marqueurs avec l'expression de la notion de devoir: le sujet se trouve dans l'état de devoir/avoir à actualiser tel ou tel prédicat. Etrangement, les linguistes qui voient dans have un marqueur de subjectivité analysent volontiers have to comme renvoyant à une obligation non assumée par l'énonciateur, simplement “constatée" (“il ne s'agit plus pour l'énonciateur de prendre en charge l'obligation qui pèse sur le sujet de l'énoncé mais de relayer une obligation extérieure et factuelle "), J. Bouscaren / J. Chuquet, $1982: 65$ ).

Dans notre modèle, la différence entre l'obligation exprimée par have to

15. He has to attend a meeting.

et l'obligation exprimée par is to

16. He is to attend a meeting.

41 découle directement des modalités de validation interlocutive caractéristiques de be et have. Par la remontée à la source qu'il prescrit, be, marqueur $\mathrm{C} 1$, présente l'obligation à laquelle est soumis le sujet en (16) comme conditionnée, en amont, par desdispositions préalables. Tandis qu'avec have to en (15), le locuteur porte à l'attention de l'interlocuteur la situation du sujet ayant à assumer l'obligation. Aucune référence n'est faite à une quelconque origine de l'obligation en question (soit que le locuteur ignore tout de l'origine soit qu'il juge non pertinente l'évocation de cette origine). L'absence de tout cadrage préalable, de toute dimension explicative, justifie le recours à have to chaque fois que, pour une raison ou pour une autre, il s'agit de contraindre l'interlocuteur à considérer non pas l'origine de la contrainte mais ses implications. Dans l'exemple suivant (17), en ayant recours à have to,la locutrice signale ainsi expressément à ses interlocuteurs que l'heure n'est plus à la discussion ${ }^{10}$ (partir ou ne pas partir). L'obligation est là, ancrée en elle (état de l'ayant) et elle l'assume (focalisation sur les conséquences) : 
17. "I simply must go away. I have to." She ran her fingers hysterically through her hair. "Don't you see that I have to go away?" (A Room with a View, E.M. Forster) [en gras dans le texte]

Pour l'expression de l'obligation, (comme pour l'expression de la possession) have peut être suivi de got. Tout en étant au passé, le verbe get permetde réintroduire, face au verbe d'état have, un procès dynamique. L'état de l'ayant se trouve ainsi, par l'intermédiaire de got, rattaché à unprocès. La forme have got s'emploiera donc dans les cas où il s'agit d'une " possession " récemment acquise et qu'on peut donc encore rattacher au procès dont elle découle. Get précédant en chronologie notionnelle have, have got évoque à la fois la “possession " et ce qui l'a précédé. C'est selon nous le seul cas où l'on peut dire qu'on a une référence (lexicale) à l'opération d'acquisition (get) qui précède l'état de “possession ". En ce qui concerne la notion d'obligation, have got s'emploiera plutôt dans un cas où il s'agit d'une obligation à caractère exceptionnel ou immédiat (dont on connaît donc encore la cause) ou, plus subtilement, pour nous maintenir dans la phase “dynamique " d'attente du passage à l'acte (ce qui peut donner lieu à des effets de sens très variés).

\section{Have a V}

43 Nous examinerons pour terminer le cas d'emploi de have dans la structure have a $V$ qui, dans certains cas, peut alterner avec le verbe simple. Dans l'article qu'elle consacre à cette construction très courante et cependant très peu étudiée (Why you can 'have a drink' when you can't *have an eat', 1988), A. Wierzbicka se fixe pour objectif d'expliquer la différence de sens entre le verbe simple et have $a V$,de façon à pouvoir prédire dans quels cas l'alternance est possible ou au contraire impossible. En dépit des efforts de l'auteure pour proposer une étude unifiée et synthétique, on reste sceptique devant la typologie qu'elle établit, fondée sur une répartition des constructions en question dans pas moins de dix sous-classes avec, à chaque nouvelle sous-classe, l'introduction de nouveaux critères. Surtout, comme nous allons le montrer, les critères retenus dans l'analyse aboutissent à des prédictions qui se révèlent pour la plupart fausses.

Le premier critère évoqué - dont découlent plus ou moins directement les autres concerne la durée du procès: tout en ayant une certaine durée (il ne doit pas être ponctuel), celui-ci doit être court (" anti-durative "). P. Cotte (1998), qui reprend pour l'essentiel les conclusions d'A. Wierzbicka (tout en proposant des explications parfois différentes), souligne de même l'incompatibilité entre la structure avec have et les verbes “suggérant par eux-mêmes une action prolongée " (d'où l'impossibilité, par exemple, de * have a watch face à have a look ).

45 L'action, dans la structure have $a V$,serait non seulement limitée dans le temps mais aussi limitée du point de vue de sa portée, son importance (" the Have $a V$ frame trivializes the importance of the action from the point of view of the agent. It presents the action as limited not only in time, but also in significance", 1988: 785). Have a try, par exemple,exprimerait une indifférence au résultat (" one who has a try seems not to care greatly "), ce que P. Cotte glose de son côté par essayer ne coûte rien", ce qui ne serait pas le cas de attempt qui lui, centré sur la réussite elle-même, est déclaré incompatible avec have.

46 Ajoutons un troisième critère essentiel aux yeux des deux auteurs : il doit toujours s'agir d'un procès bénéficiant de façon " positive" au sujet, en d'autres termes susceptible de 
lui procurer du plaisir. L'impossibilité de *have a speak face à have a chat ou a talk s'expliquerait ainsi par le fait que "speak, à la différence de chat ou de talk, ne connote pas l'agrément" (P.Cotte, 1988: 427). A. Wierzbicka cite de son côté have a kneel-down, irrecevable selon elle car, précisément, ne répondant pas à ce critère de plaisir potentiel pour le sujet, contrairement à, par exemple, have a lie-down.

On notera que ni A. Wierzbicka ni P. Cotte ne s'appuient pour leur étude de have a $V$ sur un corpus d'emplois de la-dite construction. Or, si on se fonde, comme nous l'avons fait, sur des emplois attestés, relevés dans des contextes de conversation ordinaire, on aboutit à des conclusions très différentes. En effet, comme nous allons le voir à travers les exemples qui suivent, les emplois que nous avons relevés ne répondent pas aux critères mis en avant par les auteurs cités. Tout d'abord, nous avons fréquemment rencontré have $a V$ dans des cas où le locuteur insiste sur la longueur du procès :

18. The afternoon was spent in a battle with Kevin over napping and actually taking a nap. Jesse finally got Kevin to nap by bringing him downstairs and sleeping on the couch with him. I had a really good nap with this strange dream about Jesus taking us to heaven and then kicking us out. I'm not sure why, it wasn't an unpleasant dream.

Even though I did have a long nap this afternoon, my aching head and tired body are screaming at me to go to bed.

http://www.sillypuppies.com/teri/blog/archives/2004_12.html

19. Oh Kelly, I'm so sorry you had such a bad night. Lack of sleep is a real $b^{*} t c h$, it just wrecks you the next day and makes everything else that much harder to cope with. I would be there in a flash to help if only I could! I hope Austin is tired enough today to have a LONG nap so that you can get some rest. Would your mom come and sit with the boys while you have a sleep for a few hours?? I agree with the other comments about putting Austin into bed with you if that's the only way you are both going to get some rest. Take care, sweetie - I'm thinking about you. ("long" souligné dans le texte)

http://www.kelly.typepad.com/kelly/2004/11/all_i_want_is_s.html

On notera aussi dans l'exemple ci-dessus l'emploi de have a sleep, refusé par A. Wierzbicka au motif que, contrairement à nap, d'une durée courte et donc contrôlable, la durée de sleep n'est pas prévisible.

De même, comment justifier He had a look at the file (par rapport à He looked at the file), que ce soit en arguant d'une durée courte du procès ou d'un certain désintérêt du sujet, d'une indifférence au résultat, alors que he had a look donne clairement à entendre que le référent de "he" ne s'est justement pas contenté de jeter un coup d'œil rapide sur la chemise du dossier mais qu'il en a étudié le contenu, à la recherche d'éléments précis (ce qui a forcément pris un certain temps)? Et comment rendre compte, en appliquant les critères mentionnés ci-dessus, de have a think face à think? Ne s'agit-il pas là d'un verbe qui, par lui-même, "suggère une action prolongée"? P. Cotte contourne ici la difficulté en expliquant que, à l'instar de have a try, have a think "sous-entend qu' il n'existe ni grande difficulté ni enjeu grave ". De façon générale, have est déclaré par A. Wierzbicka bannir toute idée de décision, d'initiative de la part du sujet ("When the agent merely 'has' something, the ideas of decision, initiative and planning are absent "). Dans l'extrait suivant (20), l'enjeu est des plus graves puisqu'il s'agit pour le référent du sujet "I " de réfléchir au sens de sa vie et qu'il décide d'y consacrer tout un weekend, à l'issue duquel il prend la décision de démissionner de son emploi d'assureur. C'est pourtant bien have a think qui est utilisé :

20. I did five years with an insurance company before I became a policeman, and I hated every minute of it. I didn't believe in the product, but the only way to get on 
was to sell more and it was driving me nuts. I had a long think over one weekend about what I wanted out of life, and gave in my notice on the Monday. (The Breaker, Minette Walters, 1998, Pan Books). have a look), une focalisation sur le "gain cognitif" du sujet ("mental gain ") mais en maintenant l'idée de l'absence d'un enjeu important et d'une indifférence corrélative du sujet (" the have a $V$ frame describes the action as a mental gain for the agent, but one of a rather minor caliber. The action is presented as somewhat half-hearted and only semipurposeful." $1988: 785)$. A. Wierzbicka un trait récurrent des structures en have. Plus généralement, have exprimerait une empathie avec le sujet, l'attention étant toujours portée sur l'expérience subjective de ce dernier. Ainsi s'expliquerait l'incompatibilité entre have a $V$ et les termes qualifiés de "techniques" et "scientifiques" comme ceux cités en exemple par A. Wierzbicka, converse et contemplate :

"the have a $V$ frame implies a subjective and experiential perspective; it is incompatible with the factual and objective perspective implied by technical or scientific terms, which concentrate on observable events and ignore the experiences or attitudes of the person involved ", (1988: 757).

A. Wierzbicka exclut ainsi d'emblée de son étude les verbes qualifiés de " non colloquial ", déclarés définitivement incompatibles avec la structure have a $V$. Que dire dans ce cas des exemples suivants qui emploient tous have a contemplate? Doit-on en conclure que contemplate serait devenu " subjectif"?

23. I go to Meriden moderately enough - always have a sit on the village green next to the Memorial for all the cyclists that died in the two world wars and have a contemplate". West Midlands MTB Birmingham CTC.

http://www.cyclingplus.co.uk/forum/topic.asp

24. Bob, you've confused the hell out of me, maybe that's cos I'm not reading it properly, nor am I going to at this point in time, it's way too hot and I'm tired. But I will read it properly sometime soon and have a contemplate/ [posted Nov.01, 2004].

http://www.pianoforum.net/smf/index.php 
25. Yeah maybe you are right, will have a contemplate on that one, cause the psd

file is at work, so can't change till the morning anyway.

http://www.forums.kustompcs.co.uk/showthread.php Aierzbicka, nous semble au contraire particulièrement révélatrice de leur inadéquation. P. Cotte propose une autre explication qui, pour être différente, n'en reste pas moins aussi chosiste et ne nous convainc pas davantage. Il attribue en effet l'impossibilité de *have an eat face à have a drink à une plus grande complexité du procès eat. "Un aliment solide", explique-t-il, "s'absorbe moins facilement qu'un liquide" (1998 : 428) : “ boire, c'est aspirer et avaler un liquide, opérations simples construisant vite l'entier notionnel et pouvant se succéder régulièrement (...). Manger, c'est aussi mâcher; cette opération dont la durée n'est pas totalement prévisible (elle dépend de l'aliment) empêche que les phases du procès s'enchainent régulièrement et que ce dernier soit entier notionnellement en un laps de temps court" (1998: 425). En ce qui nous concerne, il ne nous semble pas plus compliqué de manger que de boire et on ne voit pas en quoi la durée du procès drink serait plus prévisible que celle du procès eat. Tout dépend, souligne P. Cotte, de l'aliment à ingérer. Mais n'en est-il pas de même pour le liquide à absorber ?

Notre hypothèse est que, dans have $a V$, have - comme dans ses autres emplois - renvoie à un formatage particulier de la réception. Il contraint l'interlocuteur à s'intéresser aux implications de l'état de l'ayant. En d'autres termes, have garde sa valeur prospective : l'action évoquée ne peut se comprendre que par rapport à un projet beaucoup plus vaste que le but directement, naturellement associé au verbe simple. Quant à l'article $a$, nous avons montré dans d'autres études qu'il permet de déréférencer le nom ${ }^{11}$. Autrement dit, loin de renvoyer à une classification préétablie, il permet d'introduire un point de vue inédit, décalé, sur le référent. Have contribue à ce décalage dans la mesure où il oriente 
sur un projet " décalé" par rapport au but directement visé par le verbe simple (cf. have a trim of the bushes et have a walk to the post-office évoqués ci-dessus).

Dans have a drink, " $a$ " signale que le vocable "drink" ne définit que partiellement le référent et qu'en tout état de cause, il s'agit de bien autre chose que "d'absorber un liquide ". Si l'on propose à son interlocuteur de have a drink, on ne l'invite pas simplement à boire pour étancher sa soif (la question de savoir s'il a vraiment soif ou pas ne se pose d'ailleurs même pas): have a drink inclut drink mais se réfère à toute la portée, la dimension sociale de l'acte. De même, have a lie-down ne consiste pas à simplement s'allonger. On peut d'ailleurs have a lie-down en restantassis dans son fauteuil. Il s'agit, comme dans le cas de have a drink, d'une activité socialement codifiée. La pérennité de l'accord interlocutif, caractéristique de $\mathrm{C} 2$, explique, de façon générale, l'affinité des formes C2 avec l'évocation de tout ce qui relève de la norme, du stéréotype. Du verbe simple drink à la structure have a drink, on passe ainsi du fait simple consistant à boire à l'activité sociale connue et reconnue dans notre culture. Si have an eat passe difficilement auprès de la plupart des anglophones (voir cependant les occurrences attestées cidessous), have a quick eat ou have an eat out ne posent pas de problèmes :

26. I didn't get a lot of homework done [in Year 11 last year]. My father and brother had to go away and shear to bring in money. [Mother was sick]. I would go home and have a quick eat and get on the bike and go and check the dams and it takes about an hour and pull sheep out and take them home... every day. Weekends I couldn't go anywhere, so sporting or social life I had none... and it just made me too tired to come to school and I didn't want to listen to the teacher say where is your homework over and over... (Silas Marner, George Eliot, 1861)

27. Swimming worked up our appetites, so we went to have an eat out at Jusco after that, but found out it's closed on Mondays.

http://www.xanga.com/home.asp

61 En (27) out décale eat vers l'activité sociale " aller au restaurant". Have a quick eat en (26) signifie quant à lui " manger sur le pouce”. Le décalage opéré par l'article a peut aller jusqu'à faire glisser le verbe vers un sens figuré, voire métaphorique. La déréférenciation du vocable atteint un degré maximal dans des expressions du type have a lick and a promise

Dans les deux exemple suivants, c'est have an eat qui est utilisé (sans out) mais le contexte établit clairement qu'il ne s'agit pas de simplement " manger ":

28. Jeff was silent. Presently James rose. "But there's no use talking about it. I've got to be going. We have an eat tonight at Tucker's."

http://www.tanaya.net/Books/vspld10/index3.html

29. They shook hands with each other in an ecstasy of jubilation. "My God, I'm glad that's through," said the older man. "We were both fools, Barry, but thank God that horror is past. Now tell me all about everything--your trip, your plans. Let's have a good talk as we always do."

"Come on then, dad," cried Barry. "Let's have an eat first. By Jove, I feel a thousand years younger. I go to the M. O. to-morrow for an examination." http:// home.nextra.sk/dog/sitemap/bfile12414.htm

63 On notera que c'est la forme d'impératif qui confère un sens qu'il est d'usage de qualifier de “dynamique” en (29). Have n'est en soi ni dynamique ni statique, seuls les temps ou modes utilisés ainsi que le sens global de l'énoncé permettant de distinguer ces deux interprétations.

Des structures étiquetées irrecevables ou difficilement recevables dans l'article de A. Wierzbicka au motif que le procès “ ne connote pas l'agrément", comme par exemple 
have a kneel-down (qu'A. Wierzbicka compare à have a lie-down),deviennent parfaitement acceptables dès que le but du procès est décalé par rapport au but directement visé par le verbe simple, dans le cadre d'une activité normée. Ainsi have a kneel-down, qui signifie au football américain " mettre le genou au sol " pour faire tourner l'horloge sans risquer de perdre la balle. A contrario les verbes évoquant un procès pour lequel il est difficile d'imaginer un but décalé (ex : find, watch) sont difficilement concevables avec la structure have a $V$.

L'occultation du pôle source, caractéristique de have tout entier tourné vers la subséquence, rend de façon générale la structure have a $V$ difficilement compatible avec tout ce qui dénote une activité créatrice, innovante, originale et implique donc une référence au pôle source. On ne dira donc pas *have a write alors qu'on dit have a read. On notera à nouveau que si *have a write ne se dit pas, have a write-up (activité codifiée au sens “ donner son avis ", “ envoyer un commentaire ") est abondamment attestée.

Si les verbes hyperonymes (eat, speak, etc) sont rarement utilisés ${ }^{12}$ dans la structure en have, c'est parce que les activités sociales ou culturelles sont en anglais volontiers désignées par des vocables spécifiques (nibble, chew, chat, etc.). Ces vocables, comme le soulignent A. Wierzbicka et P. Cotte, sont quasiment tous monosyllabiques. Pour A. Wierzbicka, cette brièveté du radical verbal exprime la "limitation dans le temps" des procès évoqués. P. Cotte y voit quant à lui une "expression iconique, au niveau morphologique, de la marche à l'étroit signifiée par l'article ". En ce qui nous concerne, nous pensons que si l'on dit have a chat plutôt que *have a converse, c'est parce que les vocables romans n'ont pas la même liberté de recatégorisation morphosyntaxique que les vocables anglosaxons. Tout dépend du degré d'intégration du vocable au vocabulaire courant. Ainsi, attempt-aujourd'hui d'emploi plus courant en anglais que, par exemple, converse - entre-t-il aussi facilement que try dans la structure have a $V$ :

30. We're going to take an extensive look at PearPC and get back to you on it in a few months. For the time being why not have an attempt yourself by checking it out here? [publicité].

http://www.walibe.com/modules.php

31. This exercice is harder than it looks and may take some time. have an attempt and then return to it later if necessary.

http://www.iun.mb.ca/ss_m1_howtoseteffectivegoals.htm

32. However a worsening intestinal parasite was readily weakening Yanik and he was suffering terribly the next morning. We suspected that he had an E.coli infection, which could be cleared up with the powerful antibiotic Cipro (...). However during the 5-day course, one is advised not to operate heavy machinery or be exposed to direct sunlight. With enough time for only a few more days of waiting, we realized that we could not possibly have an attempt. Morally deflated, we returned to Huaraz. [2002 Mugs Stump Award Winners. Alpinisme] http://www.bdel.com/mugs_stump/2002_fb.html

67 Comme nous l'avons vu, les critères retenus par A. Wierzbicka et P. Cotte les amènent pourtant non seulement à refuser have an attempt mais, ce qui est beaucoup plus gênant, à prédire l'impossibilité de cette structure alors qu'elle est attestée et, de notre point de vue, parfaitement légitime.

Notre cadre théorique nous a permis de proposer une étude unifiée de have tout en évitant une répartition de l'analyse sur plusieurs niveaux distincts. Dans la conclusion de son article, C. Boisson (1987) insiste sur la nécessité pour l'analyse grammaticale de la “prise en compte sans confusion de trois approches connectées mais distinctes, qui sont celles de la tripartition de Morris: syntaxe, sémantique et pragmatique ". Cette 
distinction ne peut selon nous que contribuer à creuser le fossé langue/communication au lieu de nous amener à une véritable réconciliation des deux pôles. Nous proposons donc de l'abandonner au profit d'une conception de la langue comme systématique interlocutive, dont les constituants morphosyntaxiques n'ont de sens que par rapport au formatage "pragmatique" du message.

\section{BIBLIOGRAPHIE}

BENVENISTE, E. (1966). “ Etre et avoir dans leurs fonctions linguistiques ”, Problèmes de linguistique générale, 1 Paris : Gallimard

BOISSON, C. (1987). “ Anglais have, français avoir et l'empathie ”, in La Transitivité. CIEREC, Travaux LII, Université de Saint-Etienne

BOUSCAREN, J., CHUQUET, J. \& FILHOL-DUCHET, B. (1982). “ Have opérateur de localisation ” in Bouscaren, Jeanine (éd.) Cahiers de Recherche en Grammaire anglaise T.1, Gap \& Paris, Ophrys 53-72

COTTE, P. ( 1997 ). Grammaire linguistique, Didier-Erudition, CNED.

COTTE, P. (1998). “ Have n'est pas un verbe d'action : l'hypothèse de la réélaboration ”, in La transitivité, André Rousseau éd., Lille : Presses Universitaires du Septentrion

DOUAY, C. \& ROULLAND, D. (1996). “' L'absence de marque verbale en anglais dans une théorie de l'interlocution ", Travaux Linguistiques du CERLICO, Vol. 9. Rennes : Presses Universitaires de Rennes

DOUAY, C. (2000). Eléments pour une théorie de l'interlocution. Un autre regard sur la grammaire anglaise, Rennes : Presses Universitaires de Rennes, $188 \mathrm{p}$.

DOUAY, C. (2001). “ Grammar-and-Interlocution : English articles as markers of recipient role ”, Revue Québécoise de Linguistique, vol 29, n²2.

DOUAY, C. (2003). “ Des modalités de l'interlocution au système des modaux ”, CORELA -

Cognition, Représentation, Langage, vol.1, $\mathrm{n}^{\circ} 1$. Accessible en ligne à : http://revue-corela.org

JACQUES, F. (1979). Dialogiques, Paris, P.U.F.

ROULlAND, D. (2001). “Auxiliaires modaux et interlocution ”, Anglophonia 10, Toulouse : Presses Universitaires du Mirail, 93-108.

SOUESME, C. (1992) Grammaire anglaise en contexte, Gap \& Paris : Ophrys

WIERZBICKA, A. (1988). “ Why can you have a drink when you can't *have an eat? ”, in The Semantics of Grammar, Amsterdam/Philadelphia : John Benjamins.

\section{NOTES}

1. Voir Douay / Roulland (1996) et Douay (2000).

2. C. Kerbrat (1990: 6) : “L'essor actuel de l'interactionnisme [...] entraîne une mutation fondamentale de notre discipline, une révision en profondeur des principes méthodologiques, et 
même épistémologiques, qui régissent les études linguistiques ". (Les Interactions verbales, T. 1 , Paris, A. Colin).

3. Ces symboles n'ont évidemment aucune valeur intrinsèque et ne sont utilisés que pour la commodité de la présentation. Dans la mesure cependant où le système fonctionne sur un rapport source / cible, il est commode de conserver une numérotation ordonnée.

4. C'est moi qui souligne.

5. C'est moi qui souligne.

6. C'est moi qui souligne.

7. Voir dans C. Douay (2000) l'importance de la syntaxe élocutionnelle (indices prodosiques et kinésiques) dans le processus interprétatif.

8. Thèse défendue par C. Boisson (1987) que nous examinons plus loin.

9. Thèse selon laquelle avoir centre l'énoncé sur le sujet explicitement promu origine des repérages (J. Bouscaren / J. Chuquet, 1988).

10. La valeur fondamentalement injonctive de l'auxiliaire modal must, marqueur RID, maintient au contraire dans la phase d'instauration de la contrainte (C Douay, 2003 et D. Roulland, 2001).

11. Voir C. Douay : 1999 et 2000.

12. Nous avons cité plus haut des exemples attestés de have an eat. Une recherche sur le web nous a également fourni des exemples de have a speak: “ In them days, we used to have Children's Days. You go to church in the wagons and they have a trunk full of food. Then we, all the children, have a speak and sing "'. (Notre explication est que have a speak réfère ici à une activité parfaitement codifiée, dans le cadre du rituel des Journées de l'Enfant).

\section{RÉSUMÉS}

La grammaticalisation de have en anglais dans l'expression du parfait ou dans les constructions comme have to $+V$ et have $a+\mathrm{V}$ (have a drink, a look) permet de faire l'hypothèse d'une " invariance" de la valeur de ce verbe en tant que marqueur relationnel. Les explications soit en termes d'aspect, soit en termes de modalité, ne nous paraissent pas satisfaisantes dans la mesure où elles ne permettent pas une étude unifiée des emplois attestés. En particulier, les thèses selon lesquelles have renvoie à un "point de vue " nous semblent très contestables. Dans le cadre de notre recherche sur les conditions formelles de l'interlocution, have se révèle justement avoir pour fonction d'occulter toute référence à un point de vue quelconque.

Grammaticalized uses of have in perfect tenses or in constructions such as have to+ $V$ and have $a+V$ (have a drink, a look) point to a core meaning of this verb as a relational marker. Neither aspect- nor modality-based explanations seem adequate as they fail to provide a unified account of attested occurrences. In particular, emphasis on speakers' "viewpoints" should be reassessed. In the framework of our own study of the formal conditions of interlocution, we argue that the role of have is precisely to avoid any reference to any viewpoint. 
INDEX

Mots-clés : have, relation interlocutive, rôles linguistiques, décalage interlocutif, pragmatique, aspect, point de vue, empathie

\section{AUTEUR}

\section{CATHERINE DOUAY}

Université Charles-de Gaulle-Lille 3 